\title{
A non-linear Black-Scholes equation
}

\section{Yan Qiu and Jens Lorenz*}

Department of Mathematics and Statistics, University of New Mexico, Albuquerque, NM 87131, USA

E-mail: ccsindy@math.unm.edu

E-mail: lorenz@math.unm.edu

*Corresponding author

\begin{abstract}
We study a modification of the Black-Scholes equation allowing for uncertain volatility. The model leads to a partial differential equation with nonlinear dependence upon the highest derivative. Under certain assumptions, we show existence and uniqueness of a solution to the Cauchy problem.

Keywords: Black-Scholes equation; uncertain volatility; nonlinear partial differential equations.

Reference to this paper should be made as follows: Qiu, Y. and Lorenz, J. (2009) 'A non-linear Black-Scholes equation', Int. J. Business Performance and Supply Chain Modelling, Vol. 1, No. 1, pp.33-40.

Biographical notes: Yan Qiu is currently a PhD student in the Department of Mathematics and Statistics at the University of New Mexico. Her research interests are PDE approaches to option pricing and stochastic volatility.

Jens Lorenz received his $\mathrm{PhD}$ in 1975 from the University of Münster in Germany. He was a Faculty Member in Applied Mathematics at Caltech from 1983 to 1989 and joined the University of New Mexico in 1990. He is currently Interim Chair of the Department of Mathematics and Statistics. His research interests are numerical analysis and partial differential equations.
\end{abstract}

\section{Introduction}

The field of mathematical finance has gained significant attention since Black and Scholes (1973) published their Nobel Prize work in 1973. Using some simplifying economic assumptions, they derived a linear partial differential equation (PDE) of convection-diffusion type which can be applied to the pricing of options. The solution of the linear PDE can be obtained analytically.

In this paper we are interested in a non-linear modification of the Black-Scholes equation where the volatility $\sigma$ is not assumed to be constant, but is assumed to be a known function of $v_{s s}$ Here $v$ is the value of the option and $s$ is the price of the underlying asset. A simple choice for $\sigma=\sigma\left(v_{s s}\right)$, suggested for example in Wilmott (2000), is the discontinuous function 


$$
\sigma_{d}\left(v_{s s}\right)= \begin{cases}\sigma^{+} & \text {if } v_{s s}<0 \\ \sigma^{-} & \text {if } v_{s s}>0\end{cases}
$$

where $\sigma^{+}$and $\sigma^{-}$are estimates for the maximal and minimal values of the volatility, respectively. Since, the resulting PDE becomes non-linear in its highest derivative, the mathematical theory of the equation is by no means trivial. In this paper we consider the case where the volatility $\sigma$ is a smooth function of $v_{s s}$ and present a basic existence and uniqueness result.

The paper begins with a brief introduction to the classical Black-Scholes model in Section 2. Section 3 provides the derivation of the modified Black-Scholes equation. In Section 4, we sketch an existence and uniqueness proof for the Cauchy problem under periodic boundary conditions. These boundary conditions are not realistic and are used here, only to simplify the mathematical analysis of the non-linear PDE. In future work, we plan to extend our analysis and include more realistic boundary conditions. In the last section, we comment further on future research interests.

\section{Classical Black-Scholes model}

An option is an agreement that gives the holder a right, not an obligation, to buy from or sell to, the seller of the option a certain amount of an underlying asset at a specified price (the strike price) at a future time (the expiration date). Clearly, the value $v$ of an option is a function of various parameters, written $v(s, t ; \mu, \sigma ; E, T ; r)$. Here, $s$ is the price of the underlying asset; $t$ is the current time; $\mu$ is the drift of $s ; \sigma$ is the volatility of $s ; E$ is the strike price and $T$ is the expiration date of the option; $r$ is the risk-free interest rate. The assumptions leading to the classical BSM model are as follows:

1 the risk-free interest rate $r$ is a known constant for the life of the option

2 the price $s$ of the underlying asset follows a log-normal random walk and the drift $\mu$ and volatility $\sigma$ are constants known in advance

3 transaction costs associated with buying or selling the underlying asset are neglected

4 there are no dividends paid from the underlying asset

5 hedging can be done continuously

6 the price of the underlying asset is divisible so that we can trade any fractional share of the asset

7 we have an arbitrage-free market.

Let $\pi$ denote the value of a portfolio with long position in the option and short position in some quantity $\Delta$ of the underlying asset, 


$$
\pi=v(s, t)-\Delta s
$$

By assumption, the price $s$ of the underlying asset follows a log-normal random walk,

$$
d s=\mu s d t+\sigma s d X
$$

where $X$ is Brownian motion.

As time changes from $t$ to $t+d t$, the change in the value of the portfolio is due to the change in the value of the option and the change in the price of the underlying asset,

$$
d \pi=d v-\Delta d s
$$

By Ito's formula, we have

$$
d v=\left(v_{t}+\frac{1}{2} \sigma^{2} s^{2} v_{s s}\right) d t+v_{s} d s .
$$

Combining the last two equations yields

$$
d \pi=\left(v_{t}+\frac{1}{2} \sigma^{2} s^{2} v_{s s}\right) d t+\left(v_{s}-\Delta\right) d s
$$

Using a delta hedging strategy, we choose $\Delta=v_{s}$ and obtain

$$
d \pi=\left(v_{t}+\frac{1}{2} \sigma^{2} s^{2} v_{s s}\right) d t
$$

By the assumption of an arbitrage-free market, the change $d \pi$ equals the growth of $\pi$ in a risk-free interest-bearing account,

$$
d \pi=r \pi d t=r(v-\Delta s) d t
$$

Therefore,

$$
r(v-\Delta s) d t=\left(v_{t}+\frac{1}{2} \sigma^{2} s^{2} v_{s s}\right) d t .
$$

Substituting $\Delta=v_{s}$ one arrives at the Black-Scholes equation,

$$
v_{t}+r s v_{s}+\frac{1}{2} \sigma^{2} s^{2} v_{s s}-r v=0 \quad \text { for } \quad 0 \leq t \leq T
$$

The equation is supplemented by an end-condition at the expiration time $T$,

$$
v(s, T)= \begin{cases}\max (s-E, 0) & \text { for a call option; } \\ \max (E-s, 0) & \text { for a put option; } \\ H(s-E, 0) & \text { for a binary call option; } H \text { is the Heaviside function. }\end{cases}
$$


Denote the right-hand side in formula (2) by $v_{0}(s)$.

If one uses the transformation

$$
\tau=T-t, \quad x=\ln (s)+\left(r-\frac{1}{2} \sigma^{2}\right)(T-t), \quad w(x, \tau)=e^{r(T-t)} v(s, t)
$$

the equation (1) transforms to the heat equation,

$$
w_{\tau}=\frac{1}{2} \sigma^{2} w_{x x}
$$

and the end-condition (2) transforms to the initial condition:

$$
w(x, 0)=v(s, T)=v_{0}(s)=v_{0}\left(e^{x}\right)=w_{0}(x) .
$$

The problem has the explicit solution

$$
w(x, \tau)=\int_{-\infty}^{+\infty} \frac{1}{\sqrt{2 \pi \sigma^{2} \tau}} e^{-(x-y)^{2} / 2 \sigma^{2} \tau} w_{0}(y) d y
$$

or

$$
v(s, t)=e^{-r(T-t)} \int_{-\infty}^{+\infty} \frac{1}{\sqrt{2 \pi \sigma^{2}(T-t)}} e^{-\left(\ln (s)+\left(r-\frac{1}{2} \sigma^{2}\right)(T-t)-y\right)^{2} / 2 \sigma^{2}(T-t)} v_{0}\left(e^{y}\right) d y
$$

\section{Modified Black-Scholes model with variable volatility}

We can modify the assumptions leading to the Black-Scholes model in different ways regarding different parameters. Here, we focus on the constant volatility assumption. The volatility is not known in advance as a constant but is an uncertain stochastic variable. There are two traditional ways to measure volatility: implied or historical.

Another possibility is to assume a known range for the volatility $\sigma$ :

$$
0<\sigma^{-} \leq \sigma \leq \sigma^{+}
$$

where $\sigma^{+}$and $\sigma^{-}$are (estimates for) the maximal and minimal values of $\sigma$. We then have

$$
\min _{\sigma^{-} \leq \sigma \leq \sigma^{+}} \frac{1}{2} \sigma^{2} s^{2} v_{s s}= \begin{cases}\frac{1}{2}\left(\sigma^{+}\right)^{2} s^{2} v_{s s} & \text { if } v_{s s}<0 \\ \frac{1}{2}\left(\sigma^{-}\right)^{2} s^{2} v_{s s} & \text { if } v_{s s} \geq 0\end{cases}
$$

which motivates to define the discontinuous function 


$$
\sigma_{d}\left(v_{s s}\right)=\left\{\begin{array}{ll}
\sigma^{+} & \text {if } v_{s s}<0 \\
\sigma^{-} & \text {if } v_{s s}>0
\end{array} .\right.
$$

As outlined in the previous section, under delta hedging, $\Delta=v_{s}$ we have

$$
d \pi=\left(v_{t}+\frac{1}{2} \sigma^{2} s^{2} v_{s s}\right) d t
$$

Assume the minimum return on the portfolio with volatility $\sigma$ varying over the range $\sigma^{-} \leq \sigma \leq \sigma^{+}$equals the risk-free return $r \pi d t$. We then obtain

$$
\left(v_{t}+\frac{1}{2} \sigma_{d}^{2}\left(v_{s s}\right) s^{2} v_{s s}\right) d t=r \pi d t=r\left(v-s v_{s}\right) d t
$$

with $\sigma_{d}\left(v_{s s}\right)$ given by (3). One obtains the non-linear PDE

$$
v_{t}+r s v_{s}+\frac{1}{2} \sigma_{d}^{2}\left(v_{s s}\right) s^{2} v_{s s}-r v=0
$$

In this case, because of the variability of $\sigma=\sigma_{d}\left(v_{s s}\right)$, the transformation

$$
x=\ln (s)+\left(r-\frac{1}{2} \sigma^{2}\right)(T-t)
$$

applied in previous section is not useful since it depends via $\sigma$ on the solution $v$. Instead, we apply the much simpler transformation

$$
\tau=T-t, \quad x=s, \quad u(x, \tau)=e^{r \tau} v(s, t)
$$

leading to

$$
\begin{gathered}
u_{\tau}=\frac{1}{2} \sigma_{d}^{2}\left(u_{x x}\right) x^{2} u_{x x}+r x u_{x}, \quad x>0 \\
u(x, 0)=v(s, T)
\end{gathered}
$$

\section{Existence and uniqueness analysis}

The essential mathematical difficulty of (4) lies in the non-linear term $\sigma_{d}^{2}\left(u_{x x}\right) u_{x x}$. To address this essential difficulty, we consider an equation of the form

$$
u_{t}=G\left(u_{x x}\right) u_{x x}
$$

where $G: \mathbb{R} \rightarrow(0, \infty)$ is a given smooth positive function. 
The function $\sigma_{d}$ in (4) is not smooth, of course, but we can approximate $\sigma_{d}$ by a smooth function like

$$
\sigma_{\epsilon}\left(u_{x x}\right)=\frac{1}{2}\left(\sigma^{+}+\sigma^{-}\right)-\frac{1}{2}\left(\sigma^{+}-\sigma^{-}\right) \tanh \left(\frac{1}{\epsilon} u_{x x}\right), \quad \epsilon>0
$$

Figure 1 The graphs of $\sigma_{d}$ (left) and $\sigma_{\epsilon}$ (right) for $\sigma^{+}=0.3, \sigma^{-}=0.2$ and $\epsilon=0.3$ (see online version for colours)
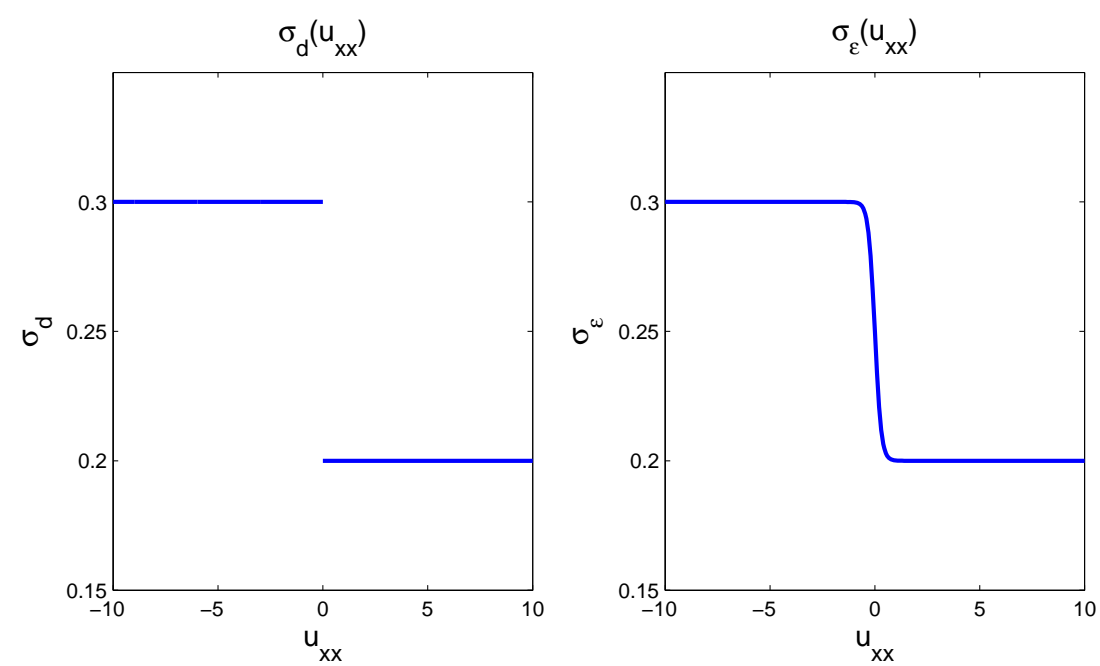

Differentiate equation (6) twice with respect to $x$ and set $w=u_{x x}$ to obtain

$$
w_{t}=h(w) w_{x x}+h^{\prime}(w) w_{x}^{2}
$$

where $h(w)=G(w)+G^{\prime}(w) w$. It will be convenient to consider the slightly more general equation

$$
w_{t}=h(w) w_{x x}+g\left(w, w_{x}\right), \quad w(x, 0)=f(x),
$$

where $h(w), g\left(w, w_{x}\right)$ and $f(x)$ are $C^{\infty}$ functions of their arguments. To concentrate on the essential mathematical difficulty, the non-linear volatility coefficient, we assume the initial function $f(x)$ to be 1-periodic; we then seek a solution $w(x, t)$, of (8) which is 1 -periodic in $x$. Other boundary conditions will be considered in future work.

Theorem 4.1 Consider the 1-periodic initial value problem (8) under the above assumptions. In addition, assume that $h(w) \geq k>0$ for all real $w$ and that $h$ and $g$ and all their derivatives are bounded functions. Then there is a unique $C^{\infty}$ solution $w(x, t)$ which is 1-periodic in $x$. The solutions exists for $0 \leq \mathrm{t}<\infty$. 
Remark: If $h$ or $g$ or their derivatives are unbounded, one can use a cut-off argument and replace $h$ or $g$ by functions $\tilde{h}$ or $\tilde{g}$ which satisfy the conditions of the theorem. For the original problem, one then obtains a result which is local in time.

To prove the existence part of the theorem, we define a sequence of functions $w^{n}(x, t)$ via the iteration

$$
w_{t}^{n+1}=h\left(w^{n}\right) w_{x x}^{n+1}+g\left(w^{n}, w_{x}^{n}\right), \quad w^{n+1}(x, 0)=f(x), \quad n=0,1,2, \ldots
$$

starting with $w^{0}(x, t) \equiv f(x)$. Since the problem (9) is linear parabolic, there is no difficulty in establishing existence, uniqueness and smoothness of the functions $w^{n}(x, t) \equiv w^{n}(x+1, t)$ for $0 \leq t<\infty$.

It can then be shown and this is the main mathematical difficulty, that the functions $w^{n}(x, t)$ are 'uniformly' smooth in any finite time interval. More precisely, for any fixed $0<T<\infty$, all derivatives are bounded independently of the iteration index $n$ :

$$
\sup _{n} \max _{x} \max _{0 \leq t \leq T}\left|\frac{\partial^{p+q}}{\partial x^{p} \partial t^{q}} w^{n}(x, t)\right| \leq C(p, q, T)<\infty
$$

General arguments as detailed in Kreiss and Lorenz (1989), for example, then show convergence of the sequence $w^{n}(x, t)$, along with all derivatives, to a smooth solution $w(x, t)$ of the problem (8).

To show uniqueness of a solution, let $\psi(x, t)$ denote the difference of two solutions. An energy estimate then yields

$$
\frac{1}{2} \frac{d}{d t}\|\psi(\cdot, t)\|_{L^{2}}^{2}=\left(\psi, \psi_{t}\right)_{L^{2}} \leq C\|\psi(\cdot, t)\|_{L^{2}}^{2}
$$

and the initial condition $\psi(x, 0) \equiv 0$ implies $\psi \equiv 0$.

\section{Future research}

In future work we plan to extend the analysis in various directions:

1 More general boundary conditions than 1-periodicity will be considered.

2 The case of a non-smooth volatility coefficient $G\left(\mu_{x x}\right)$ is of interest in applications, such as the jump function $\sigma_{d}\left(\mu_{x x}\right)$. One can treat the jump function $\sigma_{d}$ as the limit of smooth functions $\sigma_{\epsilon}$ but the limit process for the corresponding solutions is non-trivial. 
3 The case of a non-smooth volatility $\sigma_{d}\left(\mu_{x x}\right)$ can also be treated as a free boundary value problem where lines $(x(t), t)$ with $\mu_{x x}(x(t), t)=0$ will be determined as free boundaries.

\section{References}

Black, F. and Scholes, M. (1973) 'The pricing of options and corporate liabilities', J. Political Economics, Vol. 81, pp.637-654.

Kreiss, H-O. and Lorenz, J. (1989) Initial-boundary Value Problems and the Navier-stokes Equations, Academic Press, Boston.

Wilmott, P. (2000) Paul Wilmott on Quantitative Finance Volume One and Volume Two, John Wiley \& Sons Ltd, Chichester. 